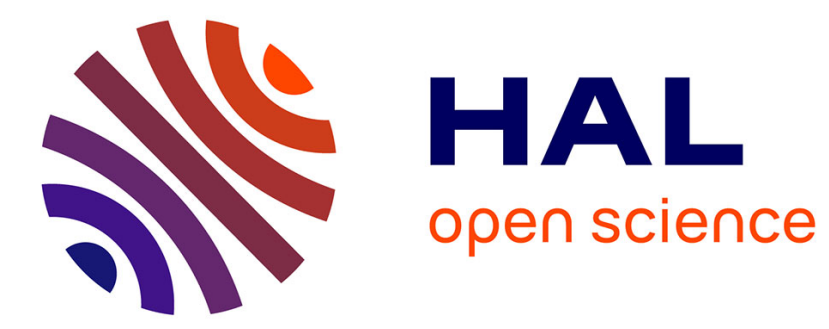

\title{
La maison solaire de Grimaud
}

\author{
J.J. Henry, X. Berger
}

\section{To cite this version:}

J.J. Henry, X. Berger. La maison solaire de Grimaud. Revue de Physique Appliquée, 1980, 15 (3), pp.569-572. 10.1051/rphysap:01980001503056900 . jpa-00244762

\section{HAL Id: jpa-00244762 https://hal.science/jpa-00244762}

Submitted on 1 Jan 1980

HAL is a multi-disciplinary open access archive for the deposit and dissemination of scientific research documents, whether they are published or not. The documents may come from teaching and research institutions in France or abroad, or from public or private research centers.
L'archive ouverte pluridisciplinaire HAL, est destinée au dépôt et à la diffusion de documents scientifiques de niveau recherche, publiés ou non, émanant des établissements d'enseignement et de recherche français ou étrangers, des laboratoires publics ou privés. 


\title{
La maison solaire de Grimaud
}

\author{
J. J. Henry et X. Berger \\ Laboratoire d'Ecothermique Solaire, C.N.R.S., Observatoire de Nice, 06 Nice, France
}

(Reçu le 6 septembre 1979, révisé le 21 novembre 1979, accepté le 26 novembre 1979)

\begin{abstract}
Résumé. - L'intégration du système solaire, en façade nord interne au moyen d'un patio, permet à la maison de Grimaud de conserver un style provençal. L'adaptation d'un stockage thermique à chaleur latente de fusion constitue une expérience originale en Europe. Le chauffage de la maison est réalisé par un flux d'air traversant une double serre. L'appoint est fourni par deux pompes à chaleur. La couverture solaire des besoins est d'environ $70 \%$ et sera portée à $75 \%$ par amélioration du système thermique.
\end{abstract}

\begin{abstract}
The provençal style of the Grimaud house is preserved by using a patio which permits solar collection on the southern side of the north wing of the building. Energy storage is done with 3.5 tons of phase change materials, which is the first real size application of such materials in Europe. The heating system is realised by a ventilation loop between the living space and a double greenhouse. Auxiliary heat is provided by two heat pumps. The results show an average $70 \%$ of the heat requirements by solar energy. Future modifications will pull this value up to $75 \%$.
\end{abstract}

1. Introduction. - La maison solaire de Grimaud est le résultat d'un échange d'idées entre les auteurs et M. Laurent Gire, maître d'œuvre à St-Tropez (Var) :

Le style provençal, bien adapté au climat local, est conservé par une forte inertie thermique du bâtiment, et l'usage d'un patio pour creer un micro-climat et abriter du vent. La maison garde un aspect traditionnel et n'est pas défigurée en façade sud par un système solaire; ce système est intégré très naturellement au moyen du patio, et ne peut être découvert qu'en rentrant à l'intérieur.

L'introduction de matériau à chaleur latente donne un aspect nouveau à la réalisation puisque la maison solaire de Grimaud est la première en Europe à utiliser un stockage de chaleur sous cette forme. Captage et stockage sont réunis au même endroit pour un maximum d'efficacité. Le système est semi-actif et utilise une gestion électronique du chauffage avec assistance de pompe à chaleur.

L'intérêt de la réalisation réside dans l'emploi de matériau à chaleur latente et dans l'intégration de manière esthétique du système solaire.

2. Description de la maison (Fig. 1). - La partie habitable a la forme d'un $\mathrm{L}$ dont les murs sont orientés sud-est et sud-ouest. Après une simulation sur ordinateur du comportement solaire, une rotation de $15^{\circ}$ vers l'ouest a été décidée pour optimiser les apports au solstice d'hiver sur la serre (ombres portées par le toit et différents arbres).

La surface habitable est de $120 \mathrm{~m}^{2}$, celle des fenêtres côté sud $18 \mathrm{~m}^{2}$. Les murs sont en béton cellulaire, les baies vitrées du séjour à double vitrage, et le taux de renouvellement d'air de $1 \mathrm{vol} / \mathrm{h}$. Le coefficient de déperditions volumiques est $1,15 \mathrm{~W} / \mathrm{m}^{3}{ }^{\circ} \mathrm{C}$.

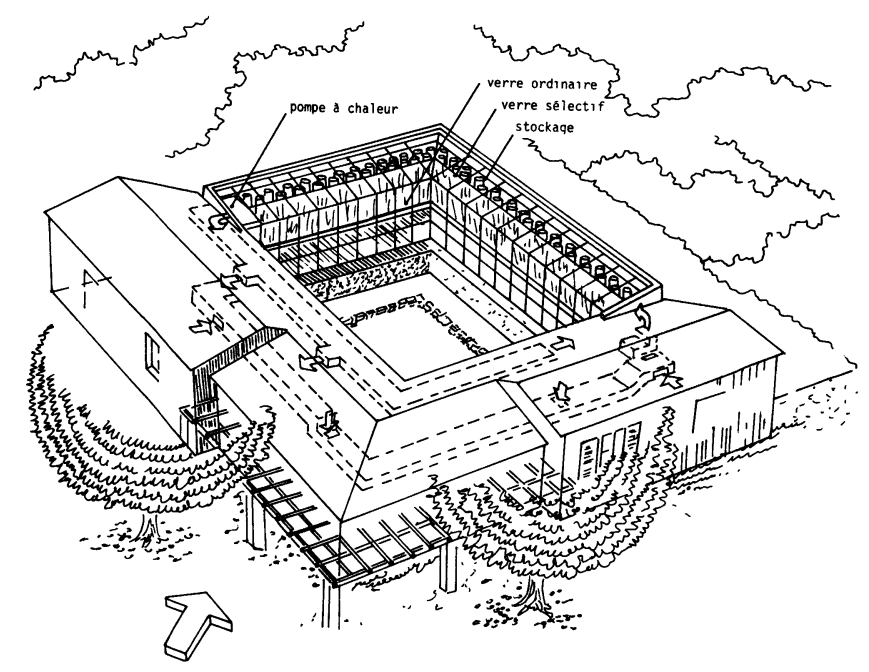

Fig. 1. - Maison de Grimaud.

[The Grimaud solar house.] 
La serre forme aussi un $\mathrm{L}$ qui complète celui de la partie habitable pour constituer le patio. Elle est enterrée et isolée côté nord. Son vitrage extérieur $\left(80 \mathrm{~m}^{2}\right)$ est ordinaire, celui intérieur, en retrait $\left(50 \mathrm{~m}^{2}\right)$, sélectif (Horti + , couche sélective vers l'intérieur). Profitant de la pente du terrain vers le sud, son niveau de base est $1,2 \mathrm{~m}$ au-dessus de celui de la partie habitable. 170 tubes de $2 \mathrm{~m}$, diamètre $10 \mathrm{~cm}$, y sont disposés verticalement et forment un échangeur de $102 \mathrm{~m}^{2}$ de surface; ils contiennent $3,5 \mathrm{t}$ de chliarolithe (réf. [1]) et permettent donc un stockage de $110 \mathrm{kWh}$. Un petit capteur a été constitué en partie haute de la serre pour fournir l'eau chaude sanitaire. Deux pompes à chaleur actionnées séparément permettent de relever les niveaux de température et d'assurer les appoints nécessaires. La circulation d'air $\left(2800 \mathrm{~m}^{3} / \mathrm{h}\right)$ en bouclage entre la maison et la serre comporte différents modes de fonctionnement selon les tempé-

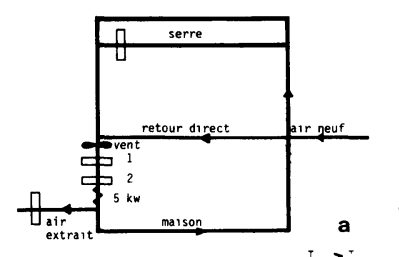

Tse
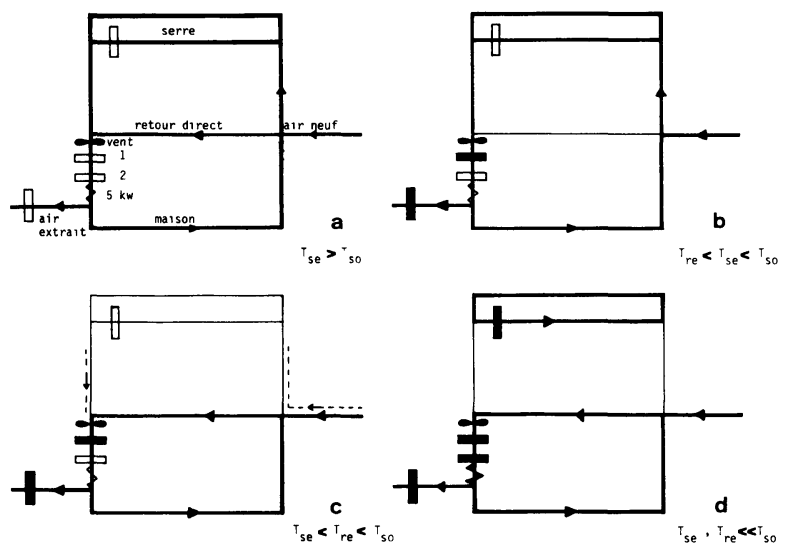

Fig. 2. - Les différents modes de fonctionnement du chauffage.

[The different working modes of the heating system.]

a) Circuit fermé sur la maison si les apports directs suffisent; sinon, passage partiel ou total par la serre si elle peut permettre d'assurer les besoins nécessaires $\left(T_{\mathrm{se}}>T_{\mathrm{so}}\right)$.

[Heating loop through the living space if the gains are sufficient. Partial circulation through greenhouse if $T_{\mathrm{se}}>T_{\mathrm{so}}$.]

b) Passage au travers de la serre, mais complément par pompe à chaleur $\mathrm{n}^{\circ} 1\left(T_{\mathrm{re}}<T_{\mathrm{se}}<T_{\mathrm{so}}\right)$.

[Circulation through the greenhouse plus heating by the heat pump $1 T_{\mathrm{re}}<T_{\mathrm{se}}<T_{\mathrm{so}}$.]

c) Circuit fermé sur la maison et chauffage par pompe $\mathrm{n}^{\circ} 1$ $\left(T_{\mathrm{se}}<T_{\mathrm{re}}<T_{\mathrm{so}}\right)$; une modification sera faite pour que l'air frais soit préchauffé par la serre.

[Heating loop through house and heat pump $1\left(T_{\mathrm{se}}<T_{\mathrm{re}}<T_{\mathrm{so}}\right)$. After a modification the new air will be preheated through the greenhouse.]

d) Dans le cas $c$ ) où la pompe no 1 ne suffit plus, la pompe no 2 extrait alors les dernières calories de la serre (circulation en boucle entre les deux parties de la serre). Si cela est insuffisant, une résistance électrique de $5 \mathrm{~kW}$ peut finalement intervenir.

[If heat pump 1 is not sufficient heat pump 2 extracts heat from the greenhouse (loop circulation between the front and the hind part of the greenhouse). If unsufficient an electric resistance of $5 \mathrm{~kW}$ is used.] ratures $\left(T_{\mathrm{re}}:\right.$ température de reprise d'air depuis la maison, $T_{\mathrm{so}}$ : température de soufflage de l'air dans la maison, $T_{\mathrm{se}}$ : température moyenne de l'air dans la serre : cas $a, b, c, d$. Ces modes sont décrits en figure 2 .

Au cours d'un cycle de réchauffage, la priorité est donnée à la ventilation de la serre aussitôt que l'on revient au cas $b$. La figure 3 montre une séquence de chauffage réelle.

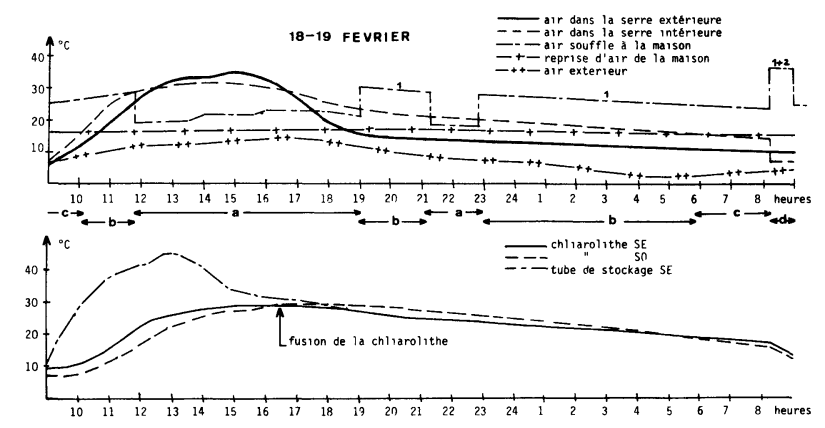

Fig. 3. - Relevé de différentes températures au cours d'un cycle de chauffage.

[Graph of registered temperature along a heating cycle.]

3. Stockage par chaleur latente de fusion. - La chliarolithe est un matériau mis au point par le Groupe Ecothermique Solaire. Il est composé principalement de :

- chlorure de calcium hexahydraté, déchet industriel provenant de la fabrication du carbonate de soude qui sert lui-même à la fabrication du verre ;

- diatomites, dont la fonction est d'éviter la ségrégation des phases de densités différentes et de maintenir en suspension les agents nucléants.

Le point de fusion est $28^{\circ} \mathrm{C}$, la chaleur de fusion $145 \mathrm{~kJ} / \mathrm{kg}$, la densité 1,52 , la chaleur spécifique $2 \mathrm{~kJ} / \mathrm{kg} \mathrm{K}$, la conductivité $0,5 \mathrm{~W} / \mathrm{m}^{\circ} \mathrm{C}$.

L'utilisation de chaleur latente à basse température permet un stockage important sur un faible intervalle de température. De ce fait, le rendement du système de captage reste toujours favorable et le volume de stockage à employer minimal.

4. Simulation sur ordinateur. - La méthode utilisée a consisté en la programmation sur ordinateur du comportement thermique de l'ensemble maison-serre, en particulier :

- calcul des ombres portées par les murs, faites, génoises et arbres sur chaque partie de la serre. Le pourcentage de surfaces éclairées à chaque instant en a été déduit ;

- évaluation des apports solaires heure par heure et mois par mois, en fonction des données météorologiques locales et pour un hiver complet;

- calcul des transferts d'énergie solaire et infrarouge, absorptions et réflexions diverses, échauffements des vitres, du matériau et de l'air. 
La modélisation du fonctionnement thermique de la maison a permis :

- de choisir la meilleure situation de la maison sur le terrain pour optimiser les apports solaires;

- d'estimer les températures à chaque instant dans les différentes parties de l'installation, et par là, d'en suivre le fonctionnement thermique;

- d'évaluer les pertes de chaque élément et donc de prévoir le bilan de fonctionnement et les améliorations possibles (vitrage sélectif, sol de serre réfléchissant).

Les résultats les plus intéressants de ces prévisions sont donnés dans le tableau I.

Tableau I. - Bilan de fonctionnement prévu pour une journée de beau temps.

[Anticipated working balance for a clear day.]

$\begin{array}{lccc} & \text { nov.-fév. } & \text { déc.-jan. } & \text { mars } \\ \begin{array}{l}\text { Contribution } \\ \text { solaire }\end{array} & 100 \% & 61 \% & 128 \% \\ \begin{array}{l}\text { Chliarolithe } \\ \text { fondue }\end{array} & 58 \% & 32 \% & 88 \%\end{array}$

Pour l'hiver complet, l'énergie solaire fournie par ventilation uniquement de la serre assure $46 \%$ des besoins de chauffage; il faut ajouter $14 \%$ obtenus par les pompes à chaleur; $10 \%$ supplémentaires proviennent enfin de l'air extrait, le reste $(30 \%)$ est par l'électricité.

5. Résultats obtenus pendant la seconde moitié de l'hiver 1978-1979. - La maison ayant été achevée fin décembre 1978, les mesures systématiques n'ont pu être entreprises qu'à partir du début février de l'année suivante. Les principaux résultats sont donnés dans les tableaux II et III.

Tableau II. - Bilan de fonctionnement observé pour une journée de beau temps.

[Observed working balance for a clear day.]

\begin{tabular}{|c|c|c|c|}
\hline & $\begin{array}{c}21-22 \text { fév. } \\
-\end{array}$ & $\begin{array}{c}8-9 \text { mars } \\
-\end{array}$ & 6-7 avr. \\
\hline $\begin{array}{r}\text { Besoins de la maison } \\
(\mathrm{kWh})\end{array}$ & 117 & 88 & 104 \\
\hline $\begin{array}{l}\text { Energie fournie par ven- } \\
\text { tilation de la serre } \\
(\mathrm{kWh})\end{array}$ & $50(41 \%)$ & $48(54 \%)$ & $93(89 \%)$ \\
\hline $\begin{array}{l}\text { Energie transférée par } \\
\text { les pompes } 1,2(\mathrm{kWh})\end{array}$ & $29(24 \%)$ & $17(20 \%)$ & $4(4 \%)$ \\
\hline Energie électrique & & & \\
\hline $\begin{array}{l}\text { - pompe } n^{\circ} 1 \\
- \text { pompe } n^{\circ} 2(\mathrm{kWh})\end{array}$ & $\begin{array}{r}31 \\
2\end{array}$ & 18 & 2 \\
\hline - ventilateur & 5 & 5 & 5 \\
\hline- total $\%$ & $35 \%$ & $26 \%$ & $7 \%$ \\
\hline
\end{tabular}

Tableau III. - Résultats par mois.

[Balance for each month.]

\begin{tabular}{|c|c|c|c|}
\hline & Février & Mars & $\begin{array}{c}\text { Avril } \\
-\end{array}$ \\
\hline $\begin{array}{l}\text { Besoins de la maison } \\
(\mathrm{kWh})\end{array}$ & 3095 & 2995 & 1150 \\
\hline $\begin{array}{l}\text { Energie fournie par } \\
\text { ventilation de la serre } \\
(\mathrm{kWh})\end{array}$ & $840(27 \%)$ & $1190(40 \%)$ & $810(71 \%)$ \\
\hline $\begin{array}{l}\text { Energie transférée par } \\
\text { les pompes } 1,2(\mathrm{kWh})\end{array}$ & $\begin{array}{l}830(27 \%) \\
210(7 \%)\end{array}$ & $\begin{array}{c}730(24 \%) \\
70(3 \%)\end{array}$ & $\begin{array}{c}130(11 \%) \\
20(2 \%)\end{array}$ \\
\hline $\begin{array}{l}\text { Energie électrique : } \\
- \text { pompe } n^{\circ} 1\end{array}$ & 925 & 770 & 120 \\
\hline - pompe $\mathrm{n}^{\circ} 2(\mathrm{kWh})$ & 140 & 45 & 16 \\
\hline - ventilateur & 150 & 190 & 55 \\
\hline - total & $39 \%$ & $33 \%$ & $16 \%$ \\
\hline
\end{tabular}

Quelques observations ont pu être faites à la suite de cette première période de fonctionnement et on en a tenu compte pour apporter des améliorations à l'installation : la pompe $\mathrm{n}^{\circ} 1$ est trop puissante. et fonctionne trop souvent; son COP est mauvais car elle agit plus sur l'air extérieur que sur l'air extrait ; elle ne permet pas une exploitation maximale de la serre. La pompe $\mathrm{n}^{\circ} 2$ a un bon COP, mais fonctionne trop rarement.

Un sol réfléchissant pour la serre externe est bénéfique $(+12 \%$ de gain), ainsi qu'il en est ressorti de l'étude théorique et d'un essai effectué pendant quelques jours.

6. Conclusions. - Les résultats déjà obtenus sont encourageants si l'on tient compte que, la maison étant juste terminée, avait encore ses murs humides; de plus elle n'était pas occupée (apports gratuits nuls) et les fenêtres étaient en permanence fermées. En tenant compte de ces éléments, on peut estimer que les prévisions sont en accord avec les résultats. Le prochain hiver permettra de fournir des résultats plus complets et correspondant au fonctionnement définitif de l'installation, dans des conditions normales d'utilisation; néanmoins on peut déjà estimer que $70 \%$ des besoins de chauffage seront satisfaits par l'énergie solaire. Cette réalisation est décrite de manière plus complète dans plusieurs documents $[2,3,4]$.

La maison de Grimaud est la première réalisation en Europe utilisant un stockage par chaleur latente de fusion. L'intégration architecturale du système solaire la distingue des autres réalisations connues. Si le surcoût solaire $(65000 \mathrm{~F})$ correspond à un amortissement en 15 ans environ, il faut lui reconnaître l'économie de $10000 \mathrm{kWh}$ qu'il permet de réaliser chaque année. De toute façon, si aucun surcoût de chauffage solaire de maison ne correspond à un amortissement inférieur à 10 ans, par contre, un confort, une sécurité et un progrès font du chauffage solaire un attrait de plus en plus observé. 


\section{Bibliographie}

[1] Schneider, M., Sylvain, J. D., Jaffrin, A., Berger, X. Brevet. Matériau accumulateur de calories à température constante et application de ce matériau. № 77/ 36.216.

[2] Berger, X., Etude simulative du comportement thermique de la maison solaire de Grimaud (publication interne, août 1978).
[3] HeNRY, J. J., La maison solaire de Grimaud (Thèse CNAM, septembre 1979).

[4] Henry, J. J. et Berger, X., Performance of the Grimaud solar house (Congrès à Izmir, en Turquie, août 1979). 